\title{
Local Electron Interaction with Point Defects in Sphalerite Zinc Selenide: Calculation from First Principles
}

\author{
O.P. MALYK ${ }^{1,2}$ and S.V. SYROTYUK ${ }^{1}$ \\ 1.-Semiconductor Electronics Department, Lviv Polytechnic National University, S. Bandera \\ Street 12, Lviv 79013, Ukraine. 2.—e-mail: omalyk@ukr.net
}

The present article deals with the description of electron scattering on the different types of point defects in zinc blende $\mathrm{ZnSe}$ on the basis of short-range principles. The electron interaction with polar and nonpolar optical phonons, piezoelectric and acoustic phonons, neutral and ionized impurities and static strain centers is considered. The electron transition probabilities and, respectively, the kinetic coefficients in zinc selenide, were calculated using the numerical eigenfunction and self-consistent potential obtained within the $a b$ initio density functional theory. The latter were evaluated using the projector augmented waves formalism as implemented in the ABINIT software suite. We investigated $\mathrm{ZnSe}$ samples with defect concentration $4.7 \times 10^{15}$ $1.08 \times 10^{17} \mathrm{~cm}^{-3}$, then calculated temperature dependencies of electron mobility and Hall factors in the range of $20-400 \mathrm{~K}$. It is shown that the theoretical curves obtained in the framework of short-range scattering models much better coincide with experimental data than the curves calculated on the basis of long-range scattering models.

Key words: Electron transport, point defects, DFT wavefunction

\section{INTRODUCTION}

In our series of previous papers, ${ }^{1-4}$ we have shown the disadvantages of the long-range models concerning the electron scattering on the point defects in II-VI and III-V semiconductors and proposed a new approach for description of the transport phenomena on the basis of short-range principles. However, in the proposed short-range approach one disadvantage remains, namely the presence of some numerical fitting parameters whose numerical values cannot be set without the availability of experimental data. On the other hand, in Ref. 5, a modification of this short-range approach was considered that enables us to eliminate the majority of the fitting parameters. The essence of this modification is to use the numerical eigenfunction and self-consistent crystal potential, obtained within the $a b$ initio density functional theory (DFT), for calculation of the electron

(Received November 8, 2017; accepted January 4, 2018; published online January 16, 2018) transition probabilities. The electron wave function and crystal self-consistent potential were obtained using projector augmented waves (PAW) as implemented in the ABINIT software. ${ }^{6}$ Attempts to describe the transport phenomena in semiconductors on the basis of the first principles are presented in the literature. ${ }^{7-11}$ However, the convergence of the proposed calculations is questionable. ${ }^{11}$ In the present article we use the sufficiently precise method proposed in Ref. 5 for calculating the kinetic coefficients of zinc selenide.

\section{THEORY}

\section{The Electron Wave Function}

The electron wave function in the conduction band, needed for the evaluation of kinetic coefficients of ZnSe crystal, is searched as a solution of the Schrödinger equation:

$$
H\left|\psi_{\alpha k}\right\rangle=E_{\alpha k}\left|\psi_{\alpha k}\right\rangle
$$

where $H$ is the Hamiltonian operator; $\psi_{\alpha \boldsymbol{k}}$ is the eigenfunction; $E_{\alpha k}$ is the energy eigenvalue, $\alpha$ is the 
Table I. The calculated band transition energies (in eV) for ZnSe crystal

\begin{tabular}{|c|c|c|c|c|c|c|}
\hline$\alpha$ & $\boldsymbol{E}$ & Grid & $\Gamma-\Gamma$ & $L-L$ & $W-W$ & $X-X$ \\
\hline 0.80 & 24 & 444 & 2.57 & 4.36 & 5.56 & 4.81 \\
\hline 0.80 & 24 & 666 & 2.58 & 4.37 & 5.57 & 4.82 \\
\hline 0.80 & 24 & 888 & 2.58 & 4.37 & 5.57 & 4.82 \\
\hline 0.80 & 36 & 444 & 2.63 & 4.38 & 5.54 & 4.77 \\
\hline 0.80 & 36 & 666 & 2.64 & 4.39 & 5.55 & 4.78 \\
\hline 0.80 & 36 & 888 & 2.64 & 4.39 & 5.55 & 4.79 \\
\hline 0.80 & 48 & 444 & 2.65 & 4.38 & 5.53 & 4.77 \\
\hline 0.80 & 48 & 666 & 2.66 & 4.39 & 5.54 & 4.78 \\
\hline 0.80 & 48 & 888 & 2.66 & 4.40 & 5.54 & 4.78 \\
\hline 0.80 & 56 & 444 & 2.66 & 4.39 & 5.53 & 4.77 \\
\hline 0.80 & 56 & 666 & 2.67 & 4.40 & 5.54 & 4.77 \\
\hline 0.80 & 56 & 888 & 2.67 & 4.40 & 5.54 & 4.78 \\
\hline 0.80 & 64 & 444 & 2.66 & 4.39 & 5.53 & 4.76 \\
\hline 0.80 & 64 & 666 & 2.67 & 4.40 & 5.54 & 4.77 \\
\hline 0.80 & 64 & 888 & 2.67 & 4.40 & 5.54 & 4.78 \\
\hline
\end{tabular}

Here $\alpha$ is the coefficient in the PBE0 hybrid exchange-correlation potential, ${ }^{17} E$ is the cutoff energy $E_{\text {cut }}$ (in Ry), and the third column presents the Monkhorst-Pack grid.

band number and $\boldsymbol{k}$ denotes the vector in the first Brillouin zone.

The oscillating electron wave function in the atom $\psi_{n}(\boldsymbol{r})$ can be represented in terms of the projector augmented waves $(\mathrm{PAW})^{12}$ :

$$
\psi_{n}(\boldsymbol{r})=\tau\left|\tilde{\psi}_{n}(\boldsymbol{r})\right\rangle,
$$

where $\tilde{\psi}_{n}(\boldsymbol{r})$ is the smooth function, and the transformation operator

$$
\tau=1+\sum_{a} \sum_{\alpha}\left(\left|\phi_{\alpha}^{a}\right\rangle-\left|\tilde{\phi}_{\alpha}^{a}\right\rangle\right)\left\langle\tilde{p}_{\alpha}^{a}\right|
$$

is based on the all-electron basis functions $\phi_{\alpha}^{a}$, pseudowave functions $\tilde{\phi}_{\alpha}^{a}$ and projector functions $\tilde{p}_{\alpha}^{a}$. In Eq. $3 \alpha$ denotes the quantum numbers, and $a$ enumerates the atom augmentation spheres.

Substituting the function defined in Eqs. 2 and 3 into the Schrödinger Eq. 1, we get a system of linear equations ${ }^{12,13}$ :

$$
\tau^{+} H \tau\left|\tilde{\psi}_{\alpha \boldsymbol{k}}\right\rangle=\tau^{+} \tau\left|\tilde{\psi}_{\alpha \boldsymbol{k}}\right\rangle \boldsymbol{E}_{\alpha \boldsymbol{k}},
$$

where $\tilde{\psi}_{\alpha k}$ is the smooth pseudowave function and the electron eigenenergies $E_{\alpha k}$ are the same as those presented in the Schrödinger Eq. 1. In Eq. $4 \tau^{+}$ defines the Hermitian conjugate of $\tau$ transformation.

It should be noted that the all-electron wave function in Eq. 1 is typically expanded in terms of $\approx$ $10^{6}$ plane waves while the pseudowave $\tilde{\psi}_{\alpha k}$, in view of its smoothness, needs only about $\approx 10^{3}$ plane waves. First, we search the pseudowave functions from the set of Eq. 4. Then we calculate the all-electron wave functions $\psi_{\alpha k}$ from an equation similar to Eq. 2.
The transformation operator $\tau$ (3) is built on the basis functions $\phi_{\alpha}^{a}, \tilde{\phi}_{\alpha}^{a}$ and $\tilde{p}_{\alpha}^{a}$. We calculated these for the atoms $\mathrm{Zn}$ and Se by means of the Atom PAW code. ${ }^{13,14}$ The PAW functions were generated for the following valence basis states: $4 s^{2} 3 d^{10}$ for $\mathrm{Zn}$ and $4 s^{2} 4 p^{4}$ for Se. The radii of the augmentation spheres $r_{\text {PAW }}$ have the following values: 1.7 and 1.8 for $\mathrm{Zn}$ and Se, respectively. The exchange and correlation effects were taken into account within the density functional theory (DFT), namely in the generalized gradient approximation (GGA) formalism suggested by Perdew, Burke and Ernzerhof (PBE) ${ }^{15}$ The output files of the AtomPAW code contain the full set of data, which are input parameters for initiation of the ABINIT code. ${ }^{6}$

The wave functions and electron energies of the ZnSe crystal were obtained by means of the ABINIT software. The experimental value of the lattice parameter $a_{0}=5.6687 \AA$ was used in the calculation. The number of the plane waves for the pseudowave function $\tilde{\psi}_{\alpha k}$ expansion was defined by the maximum value of energy $E_{\text {cut }}=64 \mathrm{Ry}$. We calculated the electronic density and crystal's Coulomb potential on the denser grid defined by limiting energy $128 \mathrm{Ry}$. Integration over the Brillouin zone was carried out on the Monkhorst-Pack $8 \times 8 \times 8$ grid. $^{16}$

In this way, we came to the conclusion that well converged results are obtained with a MonkhorstPack grid $8 \times 8 \times 8$ and limiting energies $64 \mathrm{Ry}$ and 128 Ry. A detailed convergence justification of the self-consistent electron energy spectrum calculation of the crystal $\mathrm{ZnSe}$ is presented in Table I. 


\section{Short-Range Scattering Models}

To describe the electron-phonon interaction, the wave function of the system "electron + phonon" was chosen in the form:

$$
\Psi=\psi(\boldsymbol{r}) \Phi\left(x_{1}, x_{2} \ldots x_{n}\right),
$$

where $\Phi\left(x_{1}, x_{1} \ldots x_{n}\right)$ is the wave function of the system of independent harmonic oscillators, and $\psi(\boldsymbol{r})$ is the electron wave function at the $\Gamma$ point of the Brillouin zone which was obtained from ABINIT $^{6}$ It should be noticed that the initial electron wave function $\psi(\boldsymbol{r})$ was calculated without taking into account the spin-orbit interaction; therefore, it looks like ${ }^{5}$ :

$\psi(\boldsymbol{r})=\frac{1}{\sqrt{G}} \varphi(\boldsymbol{r}) \frac{1}{\sqrt{2}}\left(\begin{array}{l}1 \\ 0\end{array}\right) \quad$ or $\quad \psi(\boldsymbol{r})=\frac{1}{\sqrt{G}} \varphi(\boldsymbol{r}) \frac{1}{\sqrt{2}}\left(\begin{array}{l}0 \\ 1\end{array}\right)$,

where the matrices $\left(\begin{array}{l}1 \\ 0\end{array}\right)$ and $\left(\begin{array}{l}0 \\ 1\end{array}\right)$ define the spinup and spin-down states, respectively. Then, using the procedure described in Ref. 1 , one can define the expression for electron-polar optical (PO) phonon transition probability:

$$
\begin{aligned}
W_{\mathrm{PO}}\left(\boldsymbol{k}, \boldsymbol{k}^{\prime}\right)= & \frac{\pi^{5} A_{\mathrm{PO}}^{2} e^{4}}{16 \varepsilon_{0}^{2} a_{0}^{8} G} \frac{M_{\mathrm{Zn}}+M_{\mathrm{Se}}}{M_{\mathrm{Zn}} M_{\mathrm{Se}}} \\
& \times\left\{\frac { 1 } { \omega _ { \mathrm { LO } } } \left[N_{\mathrm{LO}} \delta\left(E^{\prime}-E-\hbar \omega_{\mathrm{LO}}\right)+.\left(N_{\mathrm{LO}}+1\right)\right.\right. \\
& \left.\times \delta\left(E^{\prime}-E+\hbar \omega_{\mathrm{LO}}\right)\right]+\frac{2}{\omega_{\mathrm{TO}}}\left[N_{\mathrm{TO}} \delta\left(E^{\prime}-E-\hbar \omega_{\mathrm{TO}}\right)\right. \\
& \left.\left.+\left(N_{\mathrm{TO}}+1\right) \delta\left(E^{\prime}-E+\hbar \omega_{\mathrm{TO}}\right)\right]\right\}
\end{aligned}
$$

where $G$ is a number of unit cells in a crystal volume; $M_{\mathrm{Zn}}, M_{\mathrm{Se}}$ are atomic masses; $N_{\mathrm{LO}} ; N_{\mathrm{TO}}$ is the number of longitudinal (LO) and transverse (TO) phonons with a frequency $\omega_{\mathrm{LO}}$ and $\omega_{\mathrm{TO}}$, respectively; $a_{0}$ is the lattice constant; $\varepsilon_{0}$ is the dielectric constant and the following notation is introduced: $A_{\mathrm{PO}}=\int_{\Omega} \varphi^{*}\left(R^{2}-\frac{r^{2}}{3}\right) \varphi \mathrm{d} r ; R=\sqrt{3} a_{0} / 2$.

The calculation the value of integral $A_{\mathrm{PO}}$ over the volume of the unit cell was made by breaking the elementary cell into several parts, by transition from a spherical to an oblique coordinate system and, after that, by using a three-dimensional Bspline interpolation ${ }^{18}$ for calculation the values of the function $\varphi^{*} \varphi$. This approach yields the next value for the integral: $A_{\mathrm{PO}}=23.39 \times 10^{-20} \mathrm{~m}^{2}$.

Consideration of the electron-nonpolar optical (NPO) phonon interaction within the short-range approach yields the following expression for the transition probability ${ }^{5}$ :

$$
\begin{aligned}
W_{\mathrm{NPO}}\left(\boldsymbol{k}, \boldsymbol{k}^{\prime}\right) & =\frac{\pi^{3} d_{0}^{2}}{1152 a_{0}^{2} G} \frac{M_{\mathrm{Zn}}+M_{\mathrm{Se}}}{M_{\mathrm{Zn}} M_{\mathrm{Se}}} \\
& \times\left\{\frac { 1 } { \omega _ { \mathrm { LO } } } \left[N_{\mathrm{LO}} \delta\left(E^{\prime}-E-\hbar \omega_{\mathrm{LO}}\right)+\left(\mathrm{N}_{\mathrm{LO}}+1\right)\right.\right. \\
& \left.\times \delta\left(E^{\prime}-E+\hbar \omega_{\mathrm{LO}}\right)\right]+\frac{2}{\omega_{\mathrm{TO}}} \\
& \times\left[N_{\mathrm{TO}} \delta\left(E^{\prime}-E-\hbar \omega_{\mathrm{TO}}\right)+\left(\mathrm{N}_{\mathrm{TO}}+1\right)\right. \\
& \left.\left.\times \delta\left(E^{\prime}-E+\hbar \omega_{\mathrm{TO}}\right)\right]\right\}
\end{aligned}
$$

where $d_{0}=-0.371 \mathrm{eV}$ is the optical deformation potential constant.

The magnitude $d_{0}$ was calculated as an average value of three optical deformation potential constants corresponding to one longitudinal and two transverse branches of the lattice optical vibrations. The latter were evaluated using the finite displacement method ${ }^{5}$ and three-dimensional B-spline interpolation. ${ }^{18}$ The accuracy of the finite displacement method was $\sim 4 \%$ for the longitudinal branch and $\sim 4 \div 11 \%$ for transverse branches.

Using short-range principles to describe the process of the electron-acoustic phonon (AC) scattering, one can obtain the expression for transition probability for this type of interaction ${ }^{3}$ :

$$
W_{\mathrm{AC}}\left(\boldsymbol{k}, \boldsymbol{k}^{\prime}\right)=\frac{\pi^{3} k_{B} T E_{\mathrm{AC}}^{2}}{576 \hbar \mathrm{G}\left[M_{\mathrm{Zn}}+M_{\mathrm{Se}}\right]}\left(\frac{1}{c_{\|}}+\frac{2}{c_{\perp}}\right)^{2} \delta\left(E^{\prime}-E\right),
$$

where $E_{\mathrm{AC}}=-0.049 \mathrm{eV}$ is the acoustic deformation potential constant; $c_{\|}$and $c_{\perp}$ are the longitudinal and transverse sound velocity, respectively, and the elastic character of scattering is taken into account.

The magnitude $E_{\mathrm{AC}}$ was calculated as an average value of three acoustic deformation potential constants, corresponding to one longitudinal and two transverse branches of the lattice acoustic vibrations. The latter were evaluated using the finite displacement $\operatorname{method}^{5}$ and three-dimensional Bspline interpolation. ${ }^{18}$ The accuracy of the finite displacement method was $\sim 11 \%$ for the longitudinal branch and $\sim 3 \div 3.6 \%$ for transverse branches.

In Ref. 5 it was shown that in the frameworks of the short-range scattering model, the coordinate dependence of the potential energy of the electronpiezoelectric (PZ) phonon interaction has spherical symmetry and is similar to a case of $\mathrm{PO}$-scattering. This determines the value $A_{\mathrm{PZ}}=A_{\mathrm{PO}}=$ $32.83 \times 10^{-20} \mathrm{~m}^{2}$ for the integral over the volume of the unit cell. As a result, using the procedure described in Ref. 3 one can obtained the transition 


\section{Table II. Parameters of ZnSe used in calculations}

\begin{tabular}{|c|c|}
\hline Material parameter & Value \\
\hline Lattice constant, $a_{0}(\mathrm{~m})$ & $5.6687 \times 10^{-10 a}$ \\
\hline Energy gap, $E_{\mathrm{g}}(\mathrm{eV})$ & $2.825-4.84 \times 10^{-4} \mathrm{~T}^{\mathrm{b}, \mathrm{c}}$ \\
\hline Energy equivalent of the matrix element, $E_{\mathrm{p}}(\mathrm{eV})$ & $23^{\mathrm{d}}$ \\
\hline Density, $\rho\left(\mathrm{kg} \mathrm{m}^{-3}\right)$ & $5.75 \times 10^{3 \mathrm{e}}$ \\
\hline Spin-orbit splitting, $\Delta(\mathrm{eV})$ & $0.92^{\mathrm{f}}$ \\
\hline Optical deformation potential, $d_{0}(\mathrm{eV})$ & $-0.370^{\mathrm{g}}$ \\
\hline Acoustic deformation potential, $E_{\mathrm{AC}}(\mathrm{eV})$ & $-0.0491^{\mathrm{g}}$ \\
\hline Transverse optical phonon frequency, $\omega_{\mathrm{Tz}}\left(\mathrm{rad} \mathrm{s}^{-1}\right)$ & $3.88 \times 10^{13 \mathrm{~h}, \mathrm{i}}$ \\
\hline Lattice dielectric constant, $\varepsilon_{\mathrm{L}}$ & $2.0^{\mathrm{j}, \mathrm{k}}$ \\
\hline High frequency dielectric constant, $\varepsilon_{\infty}$ & $6.3^{\mathrm{k}}$ \\
\hline \multicolumn{2}{|l|}{ Elastic constants $\left(\times 10^{-11}, \mathrm{~N} \mathrm{~m}^{-2}\right)$ : } \\
\hline$C_{l}$ & $1.123-1.72 \times 10^{-4} \mathrm{~T}^{\mathrm{l}}$ \\
\hline$C_{t}$ & $0.321-0.34 \times 10^{-4} \mathrm{~T}^{\mathrm{l}}$ \\
\hline Piezoelectric tensor component, $e_{14}\left(\mathrm{C} \mathrm{m}^{-2}\right)$ & $0.049^{\mathrm{m}}$ \\
\hline
\end{tabular}

${ }^{\mathrm{a}}$ Ref. $20 .{ }^{\mathrm{b}}$ Ref. $21 .{ }^{\mathrm{c}}$ Ref. $22 .{ }^{\mathrm{d}}$ Ref. $23 .{ }^{\mathrm{e}}$ Ref. $24 .{ }^{\mathrm{f}}$ Ref. $25 .{ }^{\mathrm{g}}$ Present article. ${ }^{\mathrm{h}}$ Ref. $26 .{ }^{\mathrm{i}}$ Ref. $27 .{ }^{\mathrm{j}}$ Ref. $28 .{ }^{\mathrm{k}}$ Ref. $29 .{ }^{\mathrm{l}}$ Ref. $30 .{ }^{\mathrm{m}}$ Ref. 31.

probabilities for the electron scattering on piezoacoustic (PAC) and piezooptic (POP) phonons:

$$
\begin{aligned}
W_{\mathrm{PAC}}\left(\boldsymbol{k}, \boldsymbol{k}^{\prime}\right)= & \frac{\pi^{5} e^{2} \mathrm{e}_{14}^{2} A_{\mathrm{PZ}}^{2} k_{B} T}{128 \hbar \mathrm{G} \varepsilon_{0}^{2} \mathrm{a}_{0}^{2}\left[M_{\mathrm{Zn}}+M_{\mathrm{Se}}\right]}\left(\frac{1}{c_{\|}}+\frac{2}{c_{\perp}}\right)^{2} \\
& \times \delta\left(E^{\prime}-E\right) ; \\
W_{\mathrm{POP}}\left(\boldsymbol{k}, \boldsymbol{k}^{\prime}\right)= & \frac{\pi^{7} e^{2} e_{14}^{2} A_{\mathrm{PZ}}^{2}}{400 \varepsilon_{0}^{2} a_{0}^{4} G} \frac{M_{\mathrm{Zn}}+M_{\mathrm{Se}}}{M_{\mathrm{Zn}} M_{\mathrm{Se}}} \\
\times & \left\{\frac { 1 } { \omega _ { \mathrm { LO } } } \left[N_{\mathrm{LO}} \delta\left(E^{\prime}-E-\hbar \omega_{\mathrm{LO}}\right)+.\left(N_{\mathrm{LO}}+1\right)\right.\right. \\
\times & \left.\delta\left(E^{\prime}-E+\hbar \omega_{\mathrm{LO}}\right)\right]+\frac{2}{\omega_{\mathrm{TO}}}\left[N_{\mathrm{TO}} \delta\left(E^{\prime}-E-\hbar \omega_{\mathrm{TO}}\right)\right. \\
+ & \left.\left.\left(N_{\mathrm{TO}}+1\right) \delta\left(E^{\prime}-E+\hbar \omega_{\mathrm{TO}}\right)\right]\right\}
\end{aligned}
$$

where $e_{14}$ is the non-vanishing component of the piezoelectric tensor of the sphalerite structure and the elastic character of PAC-scattering was taken into account.

Using of the short-range approach to electron scattering on the ionized impurity (II) yields the next expression for transition probability: ${ }^{5}$

$$
W_{\mathrm{II}}\left(\boldsymbol{k}, \boldsymbol{k}^{\prime}\right)=\frac{Z_{i}^{2} e^{4} N_{\mathrm{II}} A_{\mathrm{II}}^{2} a_{0}^{6}}{512 \varepsilon_{0}^{2} \hbar V} \delta\left(E^{\prime}-E\right) ; \quad A_{\mathrm{II}}=\int_{\Omega} \Psi^{*} \frac{1}{r} \Psi \mathrm{d} \boldsymbol{r},
$$

where $N_{\text {II }}$ is the concentration of ionized impurities, and $Z_{i}$ is the multiplicity ionization of impurity. The potential energy of an electron-ionized impurity interaction is spherically symmetric, so for calculating the value $A_{\mathrm{II}}$, a method similar to $\mathrm{PO}-$ scattering was used. This yields $A_{\mathrm{II}}=0.572 \times$ $10^{-10} \mathrm{~m}^{-1}$.

Within the framework of the short-range models, the electron scattering on neutral impurity (NI) and static strain (SS) centre can be described by these expressions for transition probability ${ }^{2,5}$ :

$$
\begin{gathered}
W_{\mathrm{NI}}\left(\boldsymbol{k}, \boldsymbol{k}^{\prime}\right)=\frac{100 \pi^{2} a_{B} \hbar^{3} N_{\mathrm{NI}}}{V m^{* 2} k(E)} \delta\left(\mathrm{E}^{\prime}-E\right) ; \\
W_{\mathrm{SS}}\left(\boldsymbol{k}, \boldsymbol{k}^{\prime}\right)=\frac{2^{5} 3^{4} \pi^{3} C^{2} a_{0}^{6} e^{2} e_{14}^{2} N_{\mathrm{SS}}}{V \varepsilon_{0}^{2} \hbar} \frac{1}{q^{2}} \delta\left(E^{\prime}-E\right),
\end{gathered}
$$

where $N_{\mathrm{NI}}$ is the neutral impurity concentration; $m^{*}=\hbar^{2} k(E) \partial k(E) / \partial E ; a_{B}$ is the Bohr radius; and $C \approx 0.1 ; q=\left|\boldsymbol{k}^{\prime}-\boldsymbol{k}\right| ; N_{\mathrm{SS}}$ is the concentration of the static strain centers. In (14) $N_{\mathrm{SS}}$ is the only fitting parameter which remains, but at the present time a method for calculating the value of this concentration remains unknown.

\section{COMPARISON OF THEORY AND EXPERIMENT}

The calculated temperature dependences of electron mobility in sphalerite zinc selenide were compared with experimental data presented in Ref. 19. The Fermi level was determined from the neutrality equation for a semiconductor with donors and compensated acceptors:

$$
n+N_{\mathrm{A}}=N_{\mathrm{D}}\left[1+2 \exp \left(\frac{F-E_{\mathrm{D}}}{k_{B} T}\right)\right]^{-1},
$$

where $N_{\mathrm{D}}, N_{\mathrm{A}}, E_{\mathrm{D}}$ are the donors, acceptors concentration and donor ionization energy, respectively. When determining the Fermi level, the parameters of the ZnSe defect structure were used: $E_{\mathrm{D}}=18 \mathrm{meV} ; \quad$ sample $\mathrm{A}-N_{\mathrm{D}}=3.4 \times 10^{15} \mathrm{~cm}^{-3}$, 

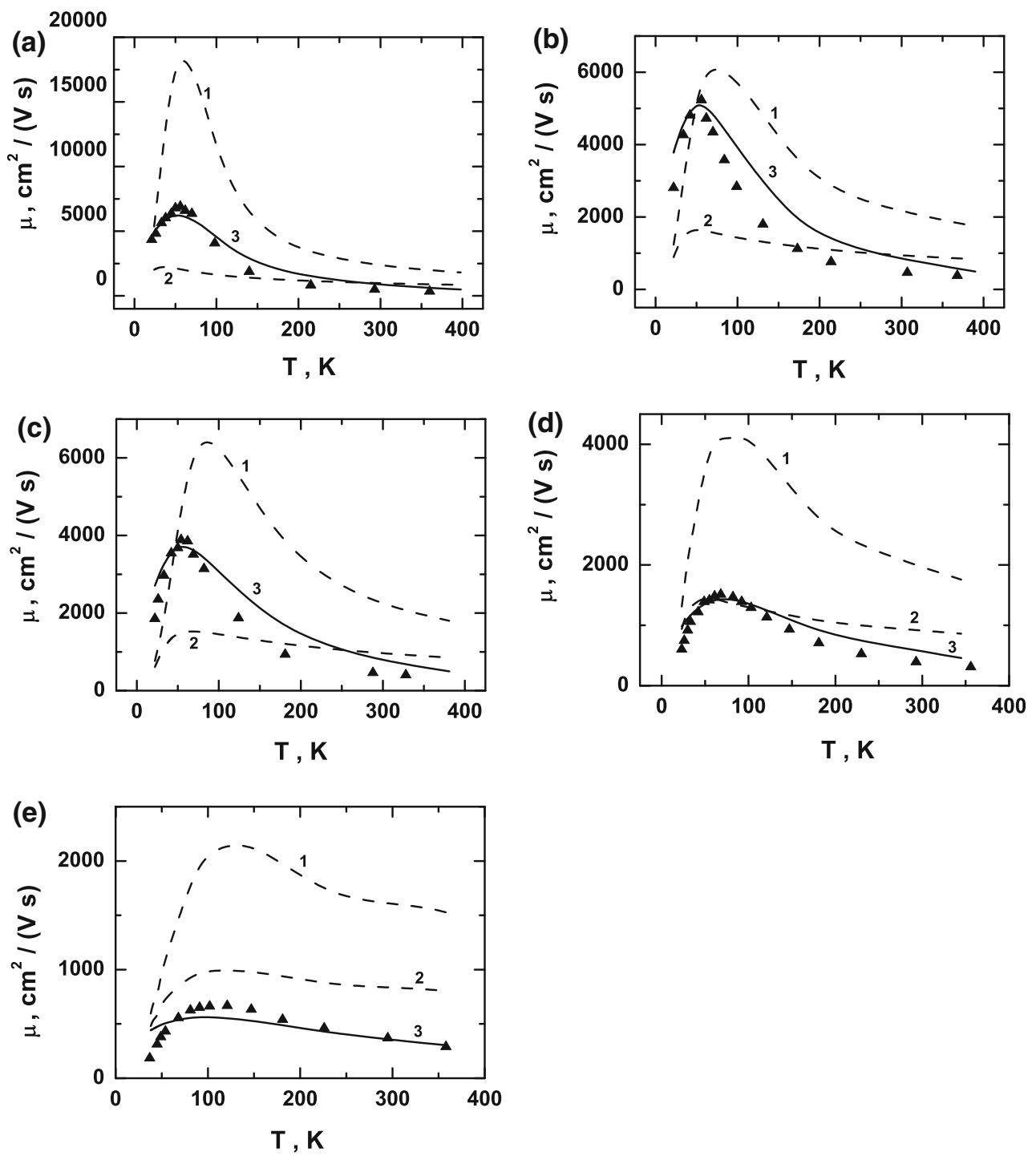

Fig. 1. Electron mobility versus temperature in zinc blende $n$-ZnSe. 1, 2-long-range scattering models (relaxation time approximation); 3-short-range scattering models. (a) sample A: $N_{\mathrm{D}}=3.4 \times 10^{15} \mathrm{~cm}^{-3} ; N_{\mathrm{A}}=1.3 \times 10^{15} \mathrm{~cm}^{-3} ; N_{\mathrm{SS}}=8.5 \times 10^{15} \mathrm{~cm}$. (b) sample B: $N_{\mathrm{D}}=1.8 \times 10^{16} \mathrm{~cm}^{-3} ; N_{\mathrm{A}}=5 \times 10^{15} \mathrm{~cm}^{-3} ; N_{\mathrm{SS}}=1.05 \times 10^{16} \mathrm{~cm}^{-3}$. (c) sample C: $N_{\mathrm{D}}=1.05 \times 10^{16} \mathrm{~cm}^{-3} ; N_{\mathrm{A}}=7.5 \times 10^{15} \mathrm{~cm}-3 ; N_{\mathrm{SS}}=1$. $5 \times 10^{16} \mathrm{~cm}^{-3}$. (d) sample D: $N_{\mathrm{D}}=3.7 \times 10^{16} \mathrm{~cm}^{-3} ; N_{\mathrm{A}}=5 \times 10^{15} \mathrm{~cm}^{-3} ; N_{\mathrm{SS}}=4.2 \times 10^{16} \mathrm{~cm}^{-3}$. (e) sample E: $N_{\mathrm{D}}=7.4 \times 10^{16} \mathrm{~cm}^{-3}$; $N_{\mathrm{A}}=3.4 \times 10^{15} \mathrm{~cm}^{-3} ; N_{\mathrm{SS}}=1.2 \times 10^{17} \mathrm{~cm}^{-3}$

$N_{\mathrm{A}}=1.3 \times 10^{15} \mathrm{~cm}^{-3} ; \quad$ sample $\quad \mathrm{B}-N_{\mathrm{D}}=1.8 \times$ $10^{16} \mathrm{~cm}^{-3}, \quad N_{\mathrm{A}}=5.0 \times 10^{15} \mathrm{~cm}^{-3} ; \quad$ sample $\mathrm{C}-N_{\mathrm{D}}=1.05 \times 10^{16} \mathrm{~cm}^{-3}, \quad N_{\mathrm{A}}=7.5 \times 10^{15} \mathrm{~cm}^{-3}$; sample $\quad \mathrm{D}-N_{\mathrm{D}}=3.7 \times 10^{16} \mathrm{~cm}^{-3}, \quad N_{\mathrm{A}}=5.0 \times$ $10^{15} \mathrm{~cm}^{-3} ; \quad$ sample $\mathrm{E}-N_{\mathrm{D}}=7.4 \times 10^{16} \mathrm{~cm}^{-3}$, $N_{\mathrm{A}}=3.4 \times 10^{16} \mathrm{~cm}^{-3}$. Parameters for zinc selenide used in our calculations are presented in Table II.

Comparisons of the theoretical curves and experimental data of the temperature dependence of electron mobility are presented in Fig. 1a-e. Solid curves 3 were calculated on the basis of the shortrange scattering models within the framework of the exact solution of Boltzmann's kinetic equation. ${ }^{32}$ Dashed curves 1 and 2 were calculated on the basis of the long-range scattering models within the framework of relaxation time approximation (see
Appendix B in Ref. 5): curve 1 corresponds to the case of low temperatures $\left(\hbar \omega>>k_{B} T\right)$, whereas curve 2 corresponds to the case of high temperatures $\left(\hbar \omega<<k_{B} T\right)$.

Comparison of theoretical curves obtained by these two approaches with experimental data shows that the short-range scattering models demonstrate much better qualitative and quantitative agreement with experiments, compared with long-range scattering models. This indicates that the short-range models more realistically describe the charge carrier scattering processes than do the long-range models.

To clarify the roles of different scattering mechanisms in Fig. $2 a$ and b, the dashed curves represent the corresponding mobility for the samples 

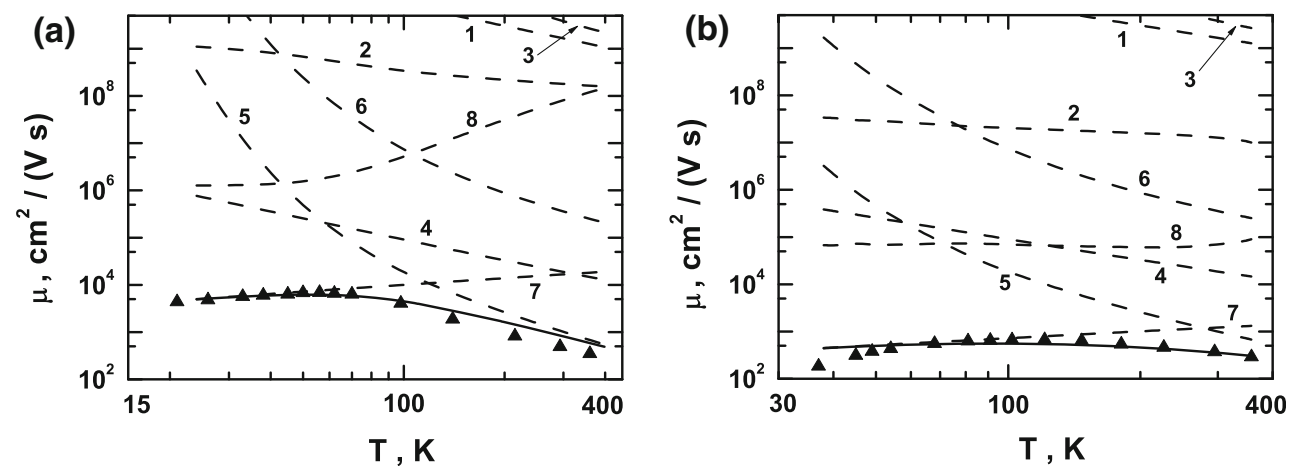

Fig. 2. The contribution of different scattering modes to electron mobility. Solid line-mixed scattering mechanism; 1,2,3,4,5,6,7,8-AC-, II-, NPO-, PAC-, PO-, POP-, SS-, NI-scattering mechanism respectively. (a) sample A; (b) sample E.

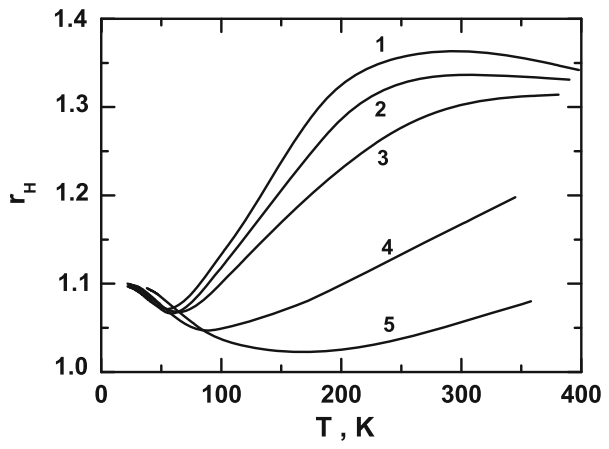

Fig. 3. The electron's Hall factor versus temperature for ZnSe crystals with different defect concentrations. 1-sample A; 2-sample B; 3-sample C; 4-sample D; 5-sample E.

with minimum and maximum defect concentration. The main scattering mechanism at low temperatures for zinc selenide is static strain scattering (curve 7). Less noticeable, but not neglected, is the role played by the piezoacoustic (curve 4) and neutral impurity (curve 8) scattering. At high temperatures the dominant scattering mechanism is polar optical phonon scattering (curve 5). It should be noted that a higher defect concentration determines the higher temperature at which the PO-scattering mechanism dominates. Other scattering mechanisms-AC-, NPO-, POP-, II-scattering-play negligibly small roles.

This temperature distribution for the scattering mechanisms determines the temperature dependence of the electron's Hall factor (see Fig. 3). It is seen that the minimums of these dependences are observed at the temperature where the transition from one scattering type (SS-scattering at low temperatures) to another scattering type (PO-scattering at high temperatures) occur. The transition temperature depends on the defect concentration: higher defect concentration determines higher transition temperature.

\section{CONCLUSIONS}

We considered electron scattering processes on the various types of crystal defects in sphalerite zinc selenide crystals on the basis of short-range principles. We calculated the transition probabilities of scattering processes using the $a b$ initio wave function and the crystal potential, obtained within the PAW formalism as implemented in the ABINIT software suite. It was established that the results obtained within the short-range models much better agree with the experimental values than those calculated on basis of the long-range models in the relaxation time approximation. We believe that the success of the approach proposed here is due to a combination of the short-range models and the allelectron self-consistent wave functions and potentials obtained on the PAW basis.

\section{REFERENCES}

1. O.P. Malyk, Comput. Mater. Sci. 33, 153 (2005).

2. O.P. Malyk, Phys. Status Solidi C 6, S86 (2009).

3. O.P. Malyk, Diamond Relat. Mater. 23, 23 (2012).

4. O.P. Malyk, Can. J. Phys. 92, 1372 (2014).

5. O.P. Malyk and S.V. Syrotyuk, Comput. Mater. Sci. 139, 387 (2017)

6. X. Gonze, F. Jollet, F. Abreu Araujo, D. Adams, B. Amadon, T. Applencourt, C. Audouze, J.-M. Beuken, J. Bieder, A. Bokhanchuk, E. Bousquet, F. Bruneval, D. Caliste, M. Cote, F. Dahm, F. Da Pieve, M. Delaveau, M. Di Gennaro, B. Dorado, C. Espejo, G. Geneste, L. Genovese, A. Gerossier, M. Giantomassi, Y. Gillet, D.R. Hamann, L. He, G. Jomard, J. Laflamme Janssen, S. Le Roux, A. Levitt, A. Lherbier, F. Liu, I. Lukačević, A. Martin, C. Martins, M.J.T. Oliveira, S. Ponce, Y. Pouillon, T. Rangel, G.-M. Rignanese, A.H. Romero, B. Rousseau, O. Rubel, A.A. Shukri, M. Stankovski, M. Torrent, M.J. Van Setten, B. Van Troeye, M.J. Verstraete, D. Waroquiers, J. Wiktor, B. $\mathrm{Xu}, \mathrm{A}$. Zhou, and J.W. Zwanziger, Comput. Phys. Commun. 205, 106 (2016)

7. K. Kaasbjerg, K.S. Thygesen, and K.W. Jacobsen, Phys. Rev. B 85, 115317 (2012).

8. O. Restrepo, K. Varga, and S. Pantelides, Appl. Phys. Lett. 94, 212103 (2009).

9. O.D. Restrepo, K.E. Krymowski, J. Goldberger, and W. Windl, New J. Phys. 16, 105009 (2014).

10. X. Li, J.T. Mullen, Z. Jin, K.M. Borysenko, M. Buongiorno Nardelli, and K.W. Kim, Phys. Rev. B 87, 115418 (2013).

11. W. Li, Phys. Rev. B 92, 075405 (2015).

12. P.E. Blöchl, Phys. Rev. B 50, 17953 (1994). 
13. N.A.W. Holzwarth, A.R. Tackett, and G.E. Matthews, Comput. Phys. Commun. 135, 329 (2001).

14. A.R. Tackett, N.A.W. Holzwarth, and G.E. Matthews, Comput. Phys. Commun. 135, 348 (2001).

15. J.P. Perdew, K. Burke, and M. Ernzerhof, Phys. Rev. Lett. 77, 3865 (1996).

16. H.J. Monkhorst and J.D. Pack, Phys. Rev. B 13, 5188 (1976).

17. P. Novák, J. Kunes, L. Chaput, and W.E. Pickett, Phys. Status Solidi B 243, 563 (2006).

18. C. de Boor, A Practical Guide to Splines (New York: Springer-Verlag, 1978), pp. 351-353.

19. S.S. Devlin, Physics and Chemistry of II-VI Compounds, ed. M. Aven and J.S. Prener (Amsterdam: North Holland, 1967), p. 418.

20. V.L. Roth, Physics and Chemistry of II-VI Compounds, ed. M. Aven and J.S. Prener (Amsterdam: North Holland, 1967), p. 97.
21. D. Theis, Phys. Status Solidi B 79, 125 (1977).

22. J. Baillou, J. Daunay, P. Bugnet, J. Daunay, C. Auzary, and R. Poindessault, J. Phys. Chem. Solids 41, 295 (1980).

23. C. Hermann and C. Weissbuch, Phys. Rev. B 15, 823 (1977).

24. W.H. Gust, J. Appl. Phys. 53, 4843 (1982).

25. M. Cardona, J. Appl. Phys. 32, 2151 (1961).

26. J.C. Irving and J. LaCombe, Can. J. Phys. 50, 2596 (1972).

27. P.A. Miles, Appl. Opt. 16, 2891 (1977).

28. G.L. House and H.G. Drickamer, J. Chem. Phys. 67, 3221 (1977).

29. A. Hadni, J. Claudel, and P. Strimer, Phys. Status Solidi 26, 241 (1968).

30. V.I. Kuskov, A.P. Rusakov, and A.M. Mentser, Sov. Phys.Sol. State 14, 1881 (1972).

31. D. Berlincourt, H. Jaffe, and L.R. Shiozawa, Phys. Rev. 129, 1009 (1963).

32. O.P. Malyk, J. Alloys Compd. 371, 146 (2004). 